Original Research Paper

\title{
Improved SDA Production in High Lipid Accumulating Strain of Mucor circinelloides WJ11 by Genetic Modification
}

\author{
${ }^{1,2 \dagger}$ Junhuan Yang, ${ }^{1 \dagger}$ Md. Ahsanul Kabir Khan, ${ }^{2}$ Sergio López-García, \\ ${ }^{1}$ Shaista Nosheen, ${ }^{1}$ Yusuf Nazir, ${ }^{1}$ Huaiyuan Zhang, ${ }^{2}$ Victoriano Garre and ${ }^{1}$ Yuanda Song \\ ${ }^{I}$ Department of Colin Ratledge Center for Microbial Lipids, School of Agricultural Engineering and Food Science, \\ Shandong University of Technology, Zibo 255000, Shandong, People's Republic of China \\ ${ }^{2}$ Department of de Genética y Microbiología (Unidad Asociada al Instituto de Química Física Rocasolano, \\ Consejo Superior de Investigaciones Científicas), Facultad de Biología, Universidad de Murcia, Murcia, 30100, Spain
}

Article history

Received: 17-01-2020

Revised: 04-03-2020

Accepted: 21-03-2020

Corresponding Author:

Yuanda Song

Colin Ratledge Center for

Microbial Lipids, School of

Agricultural Engineering and

Food Science, Shandong

University of Technology, Zibo

255000, Shandong, People's

Republic of China

Email: ysong@sdut.edu.cn

Victoriano Garre

Genética y Microbiología

(Unidad Asociada al Instituto

de Química Física Rocasolano,

Consejo Superior de

Investigaciones Científicas),

Facultad de Biología,

Universidad de Murcia

Email: vgarre@um.es

$\uparrow$ These authors contributed equally to this paper as first authors

\begin{abstract}
Stearidonic Acid (SDA; 18:4, n-3) is a $\omega-3$ polyunsaturated fatty acid which is nutritionally important and has pharmaceutical applications. Hence, scientists are trying to construct SDA producing oleaginous microorganisms by genetic modification. Two enzymes, Delta-6 Desaturase (D6D) and $\omega-3$ desaturase catalyze the reactions to produce SDA from Linoleic Acid (LA; 18:2, n-6). But the key enzyme, $\omega-3$ desaturase converts LA to ALA and GLA to SDA is absent in high lipid accumulating Mucor circinelloides WJ11, retarding its ability to produce SDA. Therefore, in this study, we overexpressed $f a d 3$ gene from Arabidopsis thaliana in M. circinelloides WJ11 to produce SDA. Overexpression of $\mathrm{fad} 3 \mathrm{gene}$ in $\mathrm{M}$. circinelloides WJ11 resulted in the production of $340 \mathrm{mg} / \mathrm{l} \mathrm{SDA}$. This research opened a new opportunity to make use of this fungus for industrial production of SDA.
\end{abstract}

Keywords: Fatty Acid Desaturase 3, SDA Production, Homologous Overexpression, Mucor circinelloides

\section{Introduction}

The fatty acids that have 18 carbon atoms or more and at least two double bonds are known as Polyunsaturated Fatty Acids (PUFAs) (Ander et al., 2003). Mammals are unable to synthesize some long chain PUFAs and, therefore, they have to be derived from Linoleic Acid (LA; 18:2, n-6) and AlphaLinolenic Acid (ALA, 18:3, n-3). The process involves a series of desaturation and elongation reactions (Wang et al., 2014). The principle source of dietary ALA is vegetable oils. ALA can be further converted to longer chain $\omega-3$ fatty acids such as Eicosapentaenoic Acid (EPA; 20:5, n-3) and Docosahexaenoic Acid (DHA, 22:5, n-3). But, the biosynthesis of EPA and DHA from ALA in human is inefficient due to the low activity of Delta-6 Desaturase (D6D) which is the rate-limiting enzyme in this process (Wang et al., 2006).

Stearidonic Acid (SDA; 18:4; n-3) occurs as a metabolic intermediate in the biosynthetic pathway to produce EPA from ALA. The requirement of D6D 
enzyme in this pathway can be bypassed by SDA. So, the oral administration of SDA is considered as an alternative way to raise the EPA level in the body tissues (Baker et al., 2016). Thus, dietary SDA was shown to have a superior activity than ALA for EPA production as it increases the EPA level in red blood cells by approximately $17 \%$, while ALA only produced an increase of about $0.1 \%$ (Harris et al., 2008).

Seafood, fish and several species of seaweed are among the marine sources of SDA where it occurs as a minor $\omega-3$ PUFA (Abeywardena et al., 2016). Among the plants, echium oil, blackcurrant seed oil and oil from the plant of Boraginaceae family are important where echium oil is the source of both $\omega-3$ and $\omega-6$ PUFA (Petrik et al., 2000; Bilgiç and Yeşilçubuk, 2012). In addition, as the yield of plantbased SDA is very low, its utilization for large scale commercial purpose will not be profitable. Nonetheless, the use of oil from fish as a commercial source of $\omega-3$ fatty acids is often controversial because of its safety concerns, shelf life limits, palatability and the possibility of overfishing (Whelan, 2009; Lu and Zhu, 2015). Therefore, due to these limitations, scientists are considering microbial lipids as a promising alternative candidate for producing SDA at commercial scale (Whelan, 2009).
SDA can also be synthesized from Gamma Linolenic Acid (GLA; 18:3, n-6), an $\omega-6$ PUFA synthesized from LA (Fig. 1) that poses significant nutritional value and medicinal benefits due to its effectiveness in treating and preventing several diseases including diabetes, cardiovascular, cancers and inflammatory disorders ( $\mathrm{Lu}$ and $\mathrm{Zhu}, 2015$; Horrobin, 1992; Fan and Chapkin, 1998; Kim et al., 2012). Mucor circinelloides (M. circinelloides) is an excellent GLA producing filamentous fungus, but unable to produce SDA because it lacks $\omega-3$ desaturase. Fatty acid Desaturase 3 (FAD3) from Arabidopsis thaliana (A. thaliana) is a $\omega-3$ desaturase which converts LA to ALA (Mendes et al., 2013) and can also be used to convert GLA to SDA (Fig. 1). Previously, we demonstrated that $M$. circinelloides can accumulated SDA by overexpressing the Delta-15 Desaturase $(D 15 D)$ gene which was cloned from Mortierella alpina (Khan et al., 2019c). However, the SDA levels were relatively low probably as a consequence of insufficient gene expression and the use of a low lipid producing strain as recipient. Therefore, in this work we have overexpressed the $A$. thaliana fad3 gene in $M$. circinelloides WJ11, a high lipid producing strain of $M$. circinelloides and constructed an SDA producing cell factory.

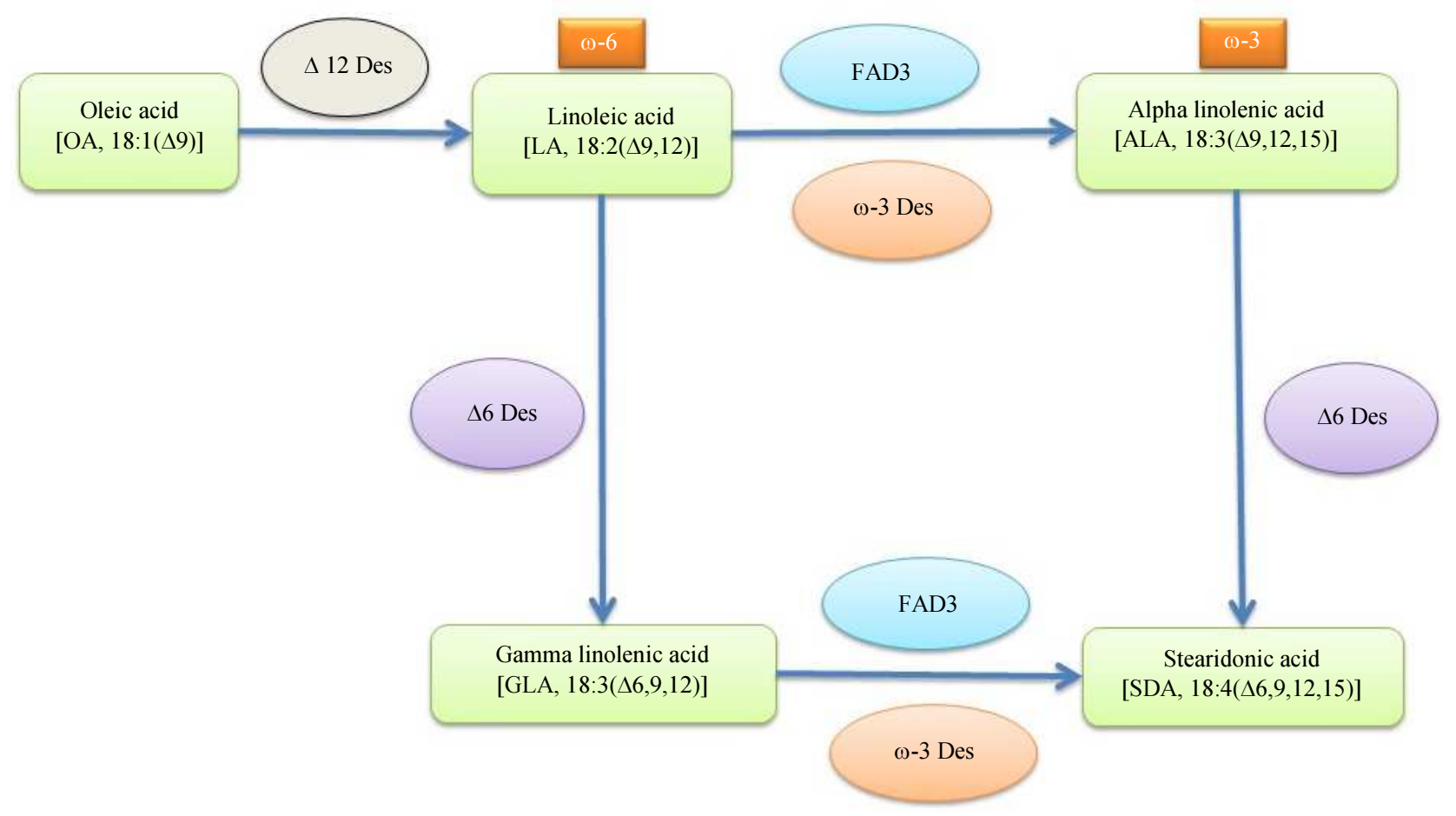

Fig. 1: Metabolic pathway of linoleic acid (LA) flux into $\omega-6$ or $\omega-3$ PUFAs to produce stearidonic acid (SDA) 


\section{Methods}

\section{Strains, Plasmids and Culture Consditions}

According to the genomic data of Arabidopsis thaliana in NCBI, the complete coding sequence of the gene fatty acid desaturase 3 ( fad3, Genebank accession number: NM 128552) is 1161 bp long. pUC19-fad3 was built by inserting the fragment of fad3 gene synthesized according to its gene sequence into pUC19 vector, which was purchased from Polepolar Biotechnology Company, Beijing. The uracil and leucine auxotrophic strain, MU760 of $M$. circinelloides WJ11 was used was used to receive the recombinant plasmid which carried the $\mathrm{fad} 3$ gene in transformation experiments. Plasmid pMAT2075 was used as the cloning and expression vector. Plasmid pMAT2079 was used as the $f a d 3$ overexpression host vector generated from pMAT2075. Escherichia coli (E. coli) DH5 $\alpha$ was used was used to maintain and propagate the recombinant plasmid. The recombinant strains Mc-2079 ( $f a d 3$ overexpressing strains) and Mc2075 (control strains) were initially cultivated in $1 \mathrm{~L}$ flasks containing $150 \mathrm{~mL} \mathrm{K \& R}$ medium for $24 \mathrm{~h}$ at $28^{\circ} \mathrm{C}$ with shaking at $130 \mathrm{rpm}$. The ingredients of this media are same as previous work (Kendrick and Ratledge, 1992) Production culture was prepared by inoculating $10 \%(\mathrm{v} / \mathrm{v})$ of the seed culture into a $2 \mathrm{~L}$ fermenter (BioFlo/CelliGen115, New Brunswick Scientific, Edison, NJ, USA) containing $1.5 \mathrm{~L}$ modified K\&R medium. This time the medium was modified because it contained $80 \mathrm{~g} / \mathrm{L}$ glucose instead of $30 \mathrm{~g} / \mathrm{L} .0 .8 \mathrm{~g} / \mathrm{L}$ of histidine was supplemented into the cultivation media when it was needed. The temperature in fermenters was maintained at $28^{\circ} \mathrm{C}$ and $\mathrm{pH}$ was maintained at 6.0 by addition of $1 \mathrm{M} \mathrm{NaOH}$ automatically, stimulated at 700 rpm with continuous aeration of $1 \mathrm{l} / \mathrm{min}$. Although the recombinant strains Mc-2079 and Mc-2075 were still leucine auxotroph (leuA $A^{-}$), it could grow in $\mathrm{K} \& \mathrm{R}$ media supplemented with $0.6 \mathrm{~g} / \mathrm{L}$ leucine.

\section{Construction of pMAT2075 and pMAT2079 Plasmids and Transformants}

To overexpress the target gene in $M$. circinelloides WJ11 a new plasmid was built and named as pMAT2075. The plasmid cloning vector pUC18 was provided by Professor Victoriano Garre (University of Murcia, Spain). The carotenogenic carRP DNA fragment which includes up- and down-stream sequences with coding regions, was amplified from the genome of M. circinelloides $\mathrm{WJ} 11$ and the primers F1/R1 used in this study were list in Table 1 . The carRP fragment was digested with SphI and SnaBI and pUC18 vector was digested with $S p h I$ and SmaI restriction endonucleases. Then the two digested DNA fragments were ligated by T4 DNA ligase. From this ligated circular vector, carRP coding sequence was deleted by doing reverse PCR using the primers F2/R2 (Additional file 1: Table 1) and linear pUC18 with carRP up- and down-stream fragments produced. This linear fragment was digested with NheI and $\mathrm{XbaI}$ restriction endonuclease. The joined pyrF and Pzrt1 fragment was amplified from plasmid PYRF using primers F3/R3 listed in Table 1 and also digested with NheI and XbaI. Then both the digested fragments were ligated by T4 DNA ligase to make pMAT2075 plasmid. Plasmid, pMAT2075, was used for the construction of recombinant plasmid carrying fad 3 gene. The newly built recombinant plasmid was named as pMAT2079. The DNA fragment of $f a d 3$ gene was amplified from the plasmid pUC19-fad3 which was used for keeping $\mathrm{fad} 3$ gene. The primers used in this case were $F A D 3-\mathrm{F} / \mathrm{R}$ which sequences are mentioned in Table 1. The recombination between linearized plasmid fragment and $f a d 3$ gene was possible due to the presence of $20 \mathrm{bp}$ homologous fragments on both sides of linearized pMAT2075 and fad3 gene fragment. The recombinant plasmid was named as pMAT2079. One step cloning kit from Takara was used to ligate the gene in the restriction endonuclease site. During the experiment of gene cloning, the ligated recombinant plasmid was maintained and propagated in chemically competent cells E. coli DH5a. The presence of recombinant plasmid in these transformants was checked by DNA sequencing. The procedure of gene cloning and the complete map of pMAT2075 and pMAT2079 were presented in Fig. 2. Both pMAT2079 and the empty plasmids pMAT2075 were digested with SmaI to release the overexpression construct and released construct was used to transform MU760 which was the uridine auxotrophic strain drived from WJ11 and the albino colonies selection was performed out as description of (Rodríguez-Frómeta et al., 2013).

\section{Gene Expression Analysis by RT-qPCR}

Cultivation of the recombinant strain of $M$. circinelloides WJ11 were done in a $2 \mathrm{~L}$ fermenter supplied with $1.5 \mathrm{~L}$ modified K\&R medium. To carry out reverse transcription-quantitative PCR (RT-qPCR) analysis, mycelium was collected at 3, 24, 48 and $72 \mathrm{~h}$. Trizol was used to extract the total RNA of $M$. circinelloides after grinding the mycelium under liquid $\mathrm{N}_{2}$. The extracted RNA was reverse transcribed by the PrimeScriptTM RT reagent kit (Takara) according to the manufacturer's instructions. The primer pair FAD3qPCR-F/R (Additional file 1: Table 1) was used to carry out RT-qPCR analysis using the in LightCycler 96 Instrument (Roche Diagnostics GmbH, Switzerland) with FastStart Universal SYBR Green Master (ROX) Supermix (Roche) as per the protocol supplied by the manufacture. The mRNA expression levels were normalized to levels of $18 \mathrm{~S}$ rRNA. The data were calculated and mRNA transcript level was determined by the method of $2^{-\Delta \Delta \mathrm{Ct}}$. The results were expressed as relative expression levels. 

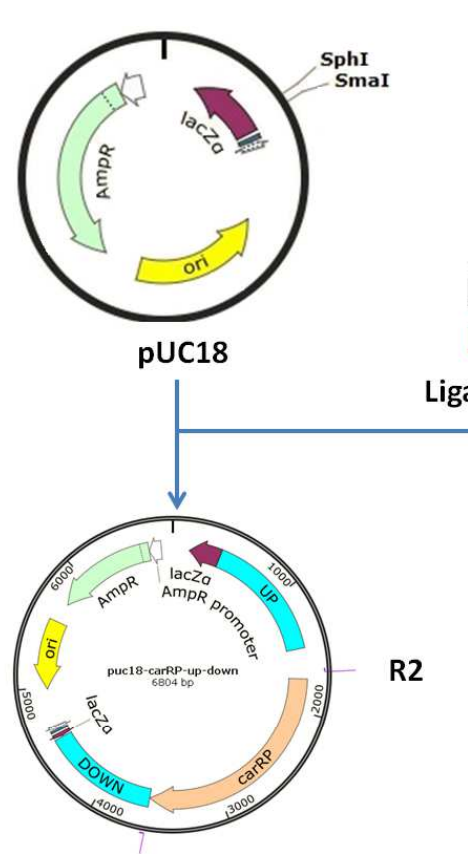

F2

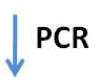

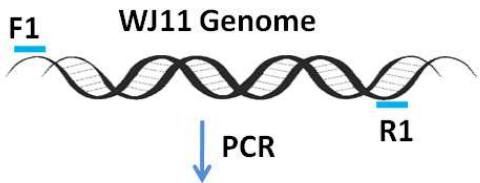

SphI

Ligation

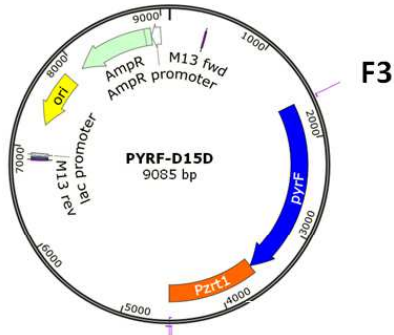

R3

$\downarrow$ PCR

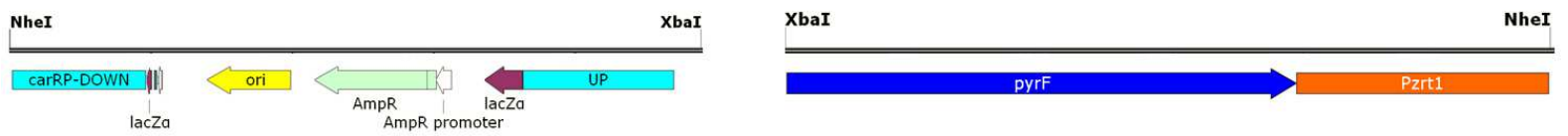

lacZa
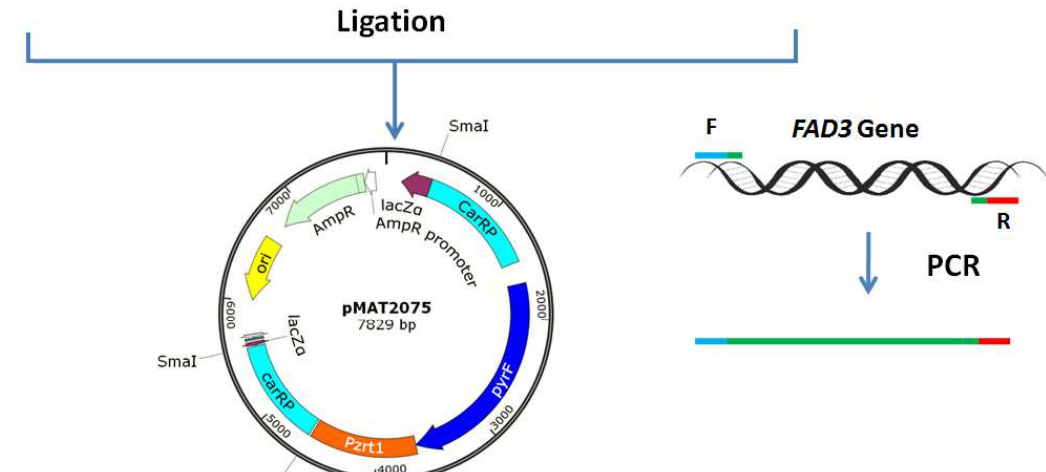

XhoI

Homologous Recombination

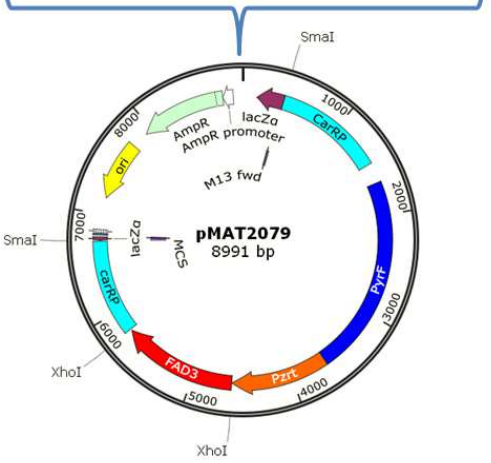

Fig. 2: Structure of plasmids pMAT2075 and pMAT2079 are shown. The fad3 gene was isolated by PCR amplification with appropriate primers. The PCR fragment was ligated into XhoI restriction site to generate plasmid as pMAT2079 
Table 1: The fatty acid composition in control and $f a d 3$ overexpressing strains

Fatty acid composition (relative $\%, w / w)$

\begin{tabular}{|c|c|c|c|c|c|c|c|}
\hline Time (hour) & $\mathrm{C}(16: 0)$ & $\mathrm{C}(18: 0)$ & $\mathrm{C}(18: 1) \mathrm{OA}$ & $\mathrm{C}(18: 2) \mathrm{LA}$ & C(18:3) GLA & C(18:3) ALA & $\mathrm{C}(18: 4) \mathrm{SDA}$ \\
\hline \multicolumn{8}{|c|}{ Mc-2075 } \\
\hline $12 \mathrm{~h}$ & $20.41 \pm 0.55$ & $6.14 \pm 0.15$ & $34.32 \pm 1.33$ & $16.51 \pm 0.65$ & $20.33 \pm 1.03$ & - & - \\
\hline $24 \mathrm{~h}$ & $23.35 \pm 0.45$ & $7.22 \pm 0.65$ & $34.61 \pm 0.42$ & $15.22 \pm 0.52$ & $14.91 \pm 0.13$ & - & - \\
\hline $36 \mathrm{~h}$ & $24.03 \pm 0.50$ & $7.48 \pm 0.35$ & $35.63 \pm 0.55$ & $14.72 \pm 0.75$ & $13.61 \pm 0.60$ & - & - \\
\hline $48 \mathrm{~h}$ & $23.89 \pm 0.40$ & $7.36 \pm 0.75$ & $36.25 \pm 0.70$ & $14.61 \pm 0.65$ & $13.35 \pm 0.35$ & - & - \\
\hline $60 \mathrm{~h}$ & $23.77 \pm 1.35$ & $7.03 \pm 0.95$ & $37.39 \pm 1.12$ & $14.73 \pm 0.75$ & $13.36 \pm 1.75$ & - & - \\
\hline $72 \mathrm{~h}$ & $23.12 \pm 0.30$ & $6.57 \pm 0.25$ & $37.03 \pm 0.33$ & $14.91 \pm 0.45$ & $13.81 \pm 0.40$ & - & - \\
\hline $84 \mathrm{~h}$ & $22.8 \pm 0.38$ & $6.40 \pm 0.32$ & $37.4 \pm 0.13$ & $15.0 \pm 0.35$ & $13.60 \pm 0.20$ & - & - \\
\hline $96 \mathrm{~h}$ & $22.45 \pm 0.20$ & $5.9 \pm 0.23$ & $37.8 \pm 0.45$ & $14.9 \pm 0.25$ & $13.40 \pm 0.40$ & - & - \\
\hline \multicolumn{8}{|l|}{ Mc-2079 } \\
\hline $12 \mathrm{~h}$ & $22.12 \pm 0.47$ & $4.76 \pm 2.11$ & $32.04 \pm 1.32$ & $14.35 \pm 1.71$ & $11.05 \pm 1.88$ & $5.76 \pm 0.93$ & $8.66 \pm 0.43$ \\
\hline $24 \mathrm{~h}$ & $21.07 \pm 0.54$ & $4.88 \pm 0.77$ & $33.20 \pm 0.64$ & $13.15 \pm 0.21$ & $12.11 \pm 0.53$ & $5.11 \pm 0.88$ & $7.48 \pm 0.47$ \\
\hline $36 \mathrm{~h}$ & $20.06 \pm 0.37$ & $4.31 \pm 0.28$ & $35.98 \pm 0.44$ & $14.23 \pm 0.34$ & $13.05 \pm 0.28$ & $4.92 \pm 0.31$ & $5.55 \pm 0.46$ \\
\hline $48 \mathrm{~h}$ & $20.74 \pm 0.99$ & $4.66 \pm 1.11$ & $36.25 \pm 0.85$ & $13.64 \pm 0.35$ & $12.01 \pm 0.50$ & $4.88 \pm 0.60$ & $5.11 \pm 0.75$ \\
\hline $60 \mathrm{~h}$ & $21.43 \pm 0.32$ & $4.87 \pm 0.35$ & $37.27 \pm 0.30$ & $13.98 \pm 0.25$ & $11.16 \pm 0.30$ & $3.47 \pm 0.25$ & $4.89 \pm 0.80$ \\
\hline $72 \mathrm{~h}$ & $21.96 \pm 0.14$ & $5.73 \pm 0.75$ & $38.18 \pm 0.50$ & $12.27 \pm 0.05$ & $10.23 \pm 0.22$ & $3.43 \pm 0.10$ & $4.66 \pm 1.00$ \\
\hline $84 \mathrm{~h}$ & $22.13 \pm 0.32$ & $5.87 \pm 0.35$ & $38.11 \pm 0.30$ & $12.98 \pm 0.25$ & $10.16 \pm 0.30$ & $3.35 \pm 0.20$ & $4.40 \pm 0.50$ \\
\hline $96 \mathrm{~h}$ & $22.76 \pm 0.14$ & $5.95 \pm 0.75$ & $37.98 \pm 0.50$ & $12.30 \pm 0.05$ & $10.12 \pm 0.22$ & $3.15 \pm 0.30$ & $4.23 \pm 0.55$ \\
\hline
\end{tabular}

The values represent the mean $\pm \mathrm{SD}$ of three independent experiments

\section{Measurement of the Glucose Concentration in Culture Medium}

Glucose oxidase Perid-test kit was used to measure the glucose concentration in the culture media following the instructions by the manufacturer (Shanghai Rongsheng Biotech Co., Ltd.). Ammonium concentration was determined by the indophenol method (Chaney and Marbach, 1962).

Determination of Cell Dry Weight, Total Lipid and Fatty Acid Profile Analysis During Fermentation Process

The samples of biomass were collected on a preweighed filter paper by filtration. And then the biomass was washed with double distilled water for three times and freeze-dried after frozen in $-80^{\circ} \mathrm{C}$ freezer for overnight. The cell dry weight was determined gravimetrically. $40 \mathrm{mg}$ dried biomass was taken for lipid extraction. The final weight of tube with total lipid was taken after extraction of total lipids with chloroform/methanol $(2: 1, \mathrm{v} / \mathrm{v})$ method.

The determination of total lipid was showed as followed equation (Khan et al., 2019a; 2019b):

$$
\text { Total } F A=\frac{T 1-T 0}{W m} \times 100
$$

Here:

$T_{1} \quad=$ Weight of tube with total lipid

$T_{0}=$ Weight of empty tube

$W m=$ weight of biomass

\section{Analysis of Fatty Acid Profile of Cell Lipid}

$20 \mathrm{mg}$ of dry mycelia was taken into a fatty acid extraction tube for the purpose of fatty acid analysis. Extraction of total lipid was done by the extraction method with chloroform/methanol $(2: 1, \mathrm{v} / \mathrm{v})$ mentioned above. $10 \%(\mathrm{v} / \mathrm{v})$ methanolic $\mathrm{HCl}$ was added to the extracted lipid and the samples were kept at $60^{\circ} \mathrm{C}$ for 3 h. Pentadecanoic acid (15:0) was added as an internal standard. The resultant fatty acid methyl esters, extracted with n-hexane, were analyzed by GC equipped with a DB-Waxetr column with $0.25 \mu \mathrm{m}$ film thickness $(30 \mathrm{~m}$ $\times 0.32 \mathrm{~mm}$ ). The GC program was set to maintain $120^{\circ} \mathrm{C}$ for $3 \mathrm{~min}$, then ramp from $120^{\circ} \mathrm{C}$ to $200^{\circ} \mathrm{C}$ at the speed of $5^{\circ} \mathrm{C} / \mathrm{min}$, then ramp from $200^{\circ} \mathrm{C}$ to $220^{\circ} \mathrm{C}$ at the speed of $4^{\circ} \mathrm{C} / \mathrm{min}$ and hold for $2 \mathrm{~min}$.

\section{Statistical Analysis}

The software SPSS 16.0 was used for statistical analysis. The calculation of mean values and the standard error of the mean were done using the data obtained from three independent experiments. Student's $t$ test was used to determine the differences between the means of the test where $P<0.05$ was considered as significantly different.

\section{Results}

Construction of fad3-Overexpression Strains of $M$. Circinelloides WJ11

To overexpress the $A$. thaliana fad3 gene in $M$. circinelloides WJ11, an expression vector pMAT2075 
was constructed to allow targeting integration of genes in the carRP locus. The pyrF gene of $M$. circinelloides WJ11 was carried by this vector. This gene encodes orotidine 5'phosphate decarboxylase and is a selectable marker for $M$. circinelloides WJ11. The strong promoter zrt 1 worked for fad3 gene overexpression. 1-kb sequences upstream and downstream of the carotenogenic carRP gene flanked the previous elements to permit chromosomal integration by the whole DNA fragment construct through homologous recombination (Zhang et al., 2017) (Fig. 2). Gene replacement in the carRP local resulted in albino colonies that are recognizable among the yellow wild-type colonies. The fad 3 cDNA with codon optimization for $M$. circinelloides was cloned in pMAT2075 producing plasmid pMAT2079. Transformants were generated by transferring the overexpression construct digested with SmaI from pMAT2079, recombinant plasmid and pMAT2075, the empty plasmid. After X vegetative cycles the transformants were obtained from plasmid pMAT2075 and pMAT2079 respectively that showed homogenous albino color, suggesting they were homokaryons for the integration in the carRP locus. The integration of target gene in transformants were confirmed by doing PCR. The primer pair checking $\mathrm{F} / \mathrm{R}$, listed in Table 1 , was used to amplify the DNA fragment for this purpose. The primers designed in such a way that it could amplify the fragment from carRP $1 \mathrm{~kb}$ upstream to carRP $1 \mathrm{~kb}$ downstream of genomic DNA, therefore, amplified a 5377- and 6643-bp (including gene) sequence from Mc-2075 and Mc-2079 respectively. The size of the PCR products from the two transformants obtained with pMAT2079 and the one obtained with pMAT2075 showed the expected sizes, confirming that the fad3 overexpressing construct had been integrated into the genome of the transformants. The two transformants harboring $\mathrm{fad} 3$ gene were named as Mc-2079 and Mc-2079-1 and the control transformant without this gene was named as Mc-2075 (Fig. 3). Additional screening was carried out (data was not mentioned) and only the higher lipid accumulation strain (Mc-2079) was selected for further study.

\section{Fad3 gene Expression Analysis in the Overexpressing Transformant}

The mycelia were harvested at 3, 24, 48 and $72 \mathrm{~h}$ of culture time in $2 \mathrm{~L}$ fermenter with $1.5 \mathrm{~L} \mathrm{K \& R}$ medium to perform the Real-time quantitative PCR to analyze the mRNA levels of fad3 gene in recombinant strain (Fig. 4). The mRNA expressing levels of fad3 gene from Mc-2079 were regarded as 1 at $3 \mathrm{~h}$ and in comparison, with it, fad 3 gene expression levels at other time were determined. It was observed that the expression level increased quickly from 3 to $24 \mathrm{~h}$ and gradually decreased with after that time. These results reveal that $f a d 3$ mRNA expression was found at high levels during the whole fermentation process. These observations indicate the overexpression of $f a d 3$ gene in the recombinant strain.

\section{Effect on Cell Growth and Lipid Accumulation in fad3 Over-Expression Transformant}

In the recombinant strain Mc-2079, the effect of $\mathrm{fad} 3$ gene over-expressed in transformant on cell growth and lipid accumulation has been investigated and was shown in Fig. 5. This figure also highlights the rate of utilization of ammonium and glucose in the culture medium. During the entire bioprocess, adequate concentrations of glucose remained in excess (Fig. 5a), but ammonium was depleted at approximately $24 \mathrm{~h}$ by Mc-2079 (Fig. 5b). CDW was found to be increased drastically from $9 \mathrm{~h}$ of cultivation and slowed down gradually after the depletion of nitrogen (Fig. 5c). The fungus started to produce lipids from the culture medium immediately after nitrogen exhaustion. The total lipid increased quickly from $12 \mathrm{~h}$, reached at the peak level at $72 \mathrm{~h}$ and then gradually slowed down. It was found that the highest content of TFAs was $35.50 \%$ in Mc-2079 (Fig. 5d).
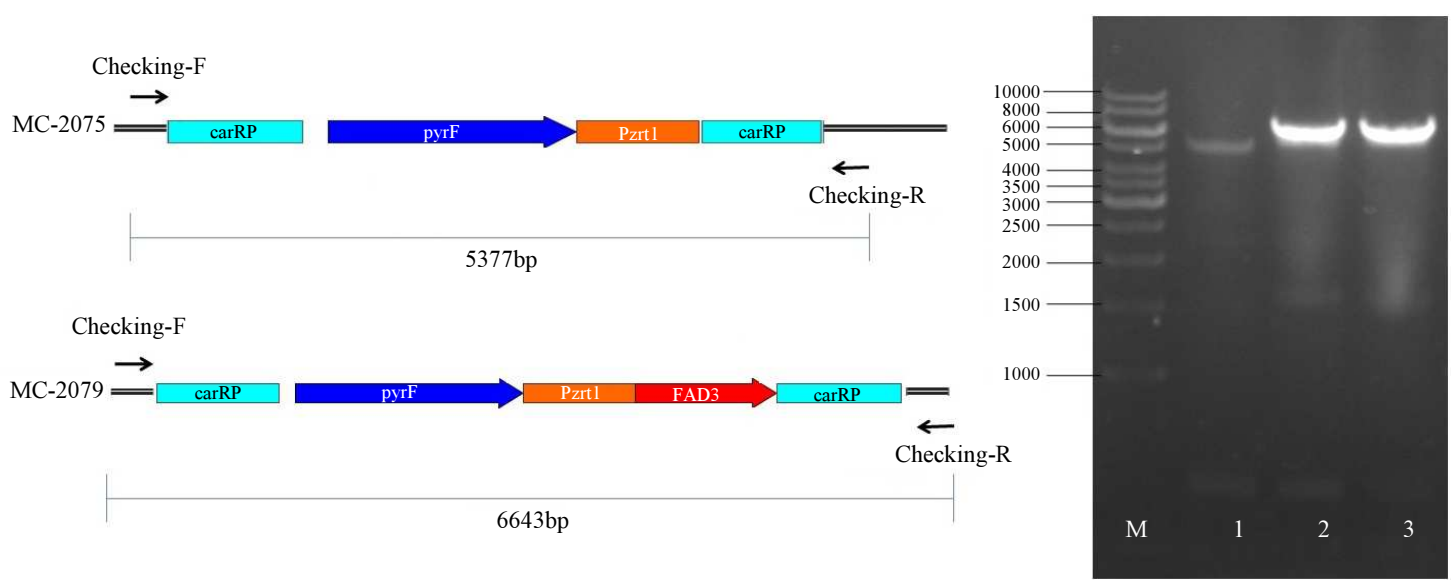

Fig. 3: PCR amplification of genome of control and recombinant strains with the primers checking-F/R. Lane 1 shows the result using DNA from the control strain Mc-2075 and lane 2 and 3 show the results using DNA from the recombinant strains Mc-2079 and 


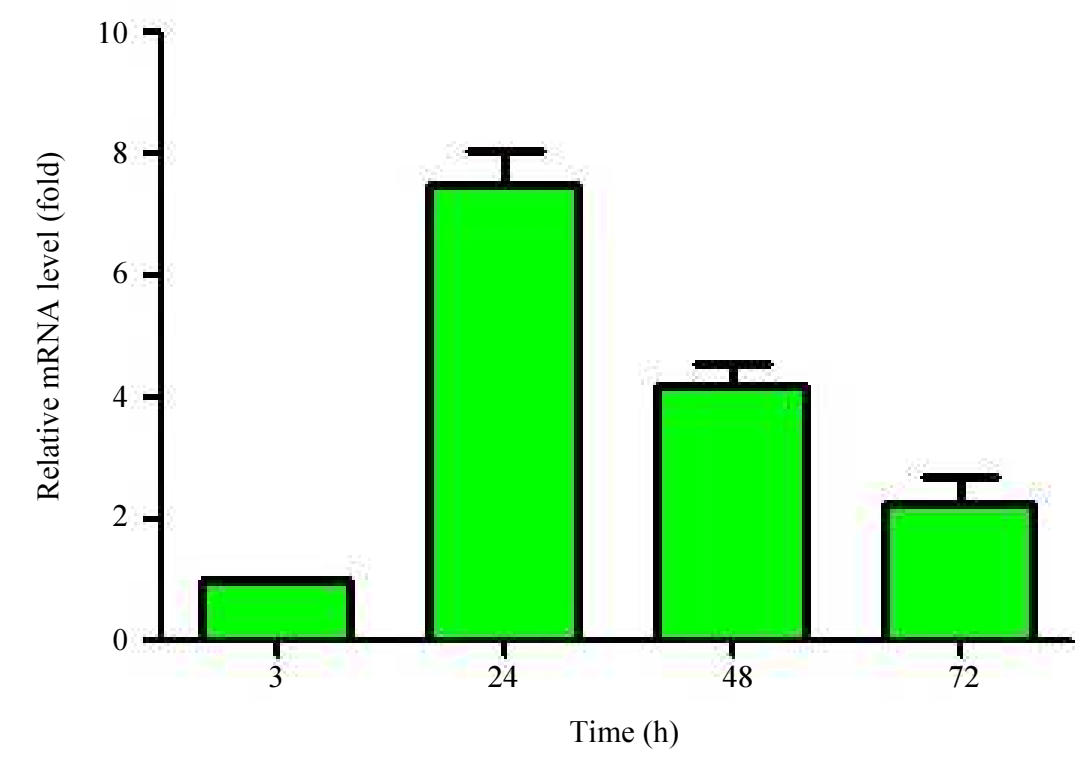

Fig. 4: Determination of expression levels of $f a d 3$ gene by RT-qPCR in the overexpressing strains
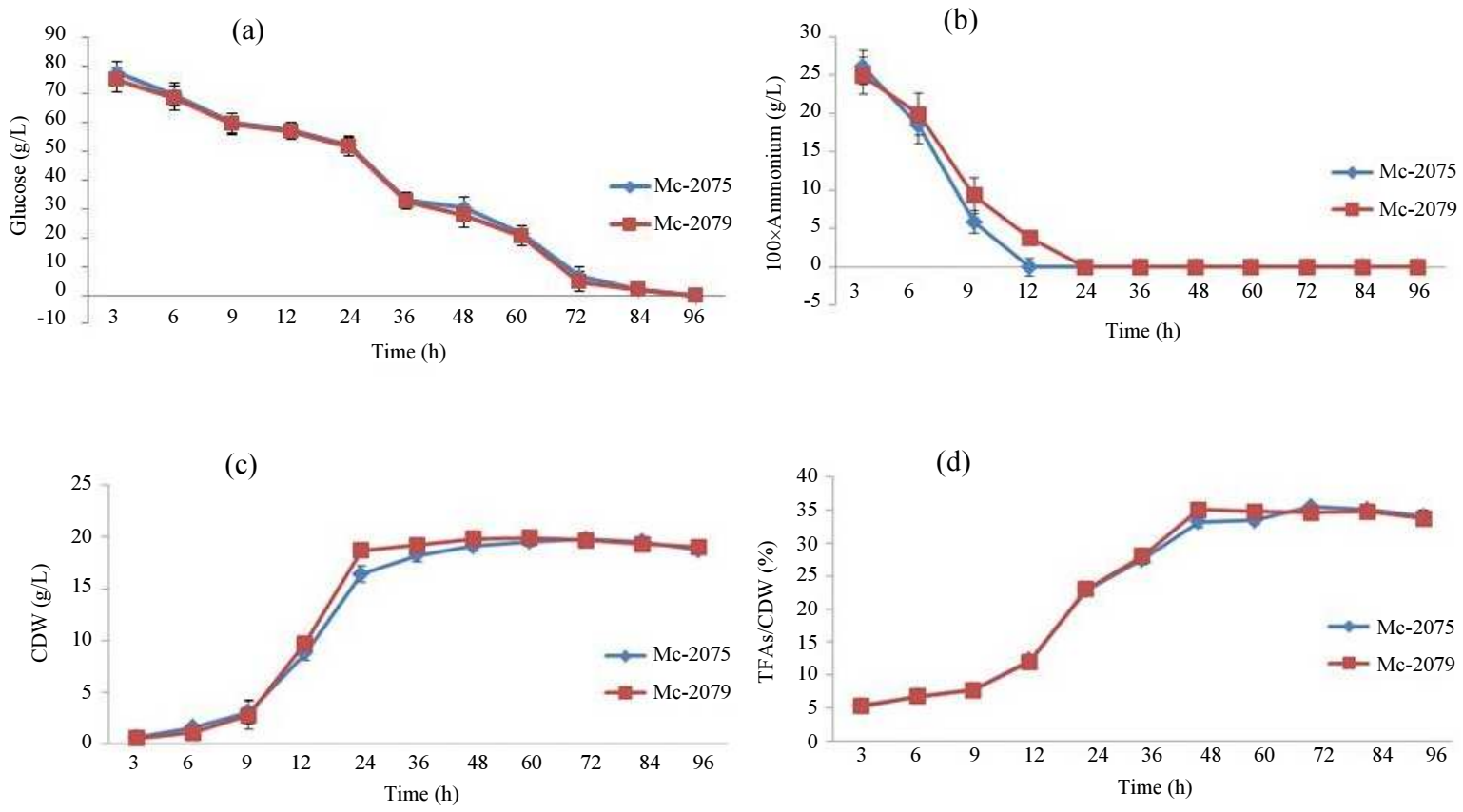

Fig. 5: Cell growth and lipid accumulation of $f a d 3$ overexpressing strains. Recombinant Mc-2079 and control strain Mc-2075 cultures were grown in $1.5 \mathrm{~L}$ modified $K$ and $R$ medium and (a) Glucose concentration, (b) Ammonium concentration, (c) Cell Dry Weight (CDW) and (d) Lipid content was measured. Samples from the fermenter were taken at the indicated times. The values were mean of three biological replicates. Error bars represented the standard error of the mean

\section{SDA Accumulation in Recombinant $M$.} Circinelloides

In the fad 3 overexpressing strains, SDA percentages were found higher at the beginning and there was a decreasing trend with increase of time (Table 1).

But the total SDA yield started to increase with progress of time and reached at maximum level at $60 \mathrm{~h}$ and then decreased gradually. Total SDA yield was calculated based on the TFAs content (Table 2).

The maximum SDA yields of $340 \mathrm{mg} / \mathrm{L}$ were detected in the recombinant strains. Meanwhile, other fatty acid contents had a little change in the recombinant fungi in comparison to the same fatty acids accumulated by the control strain (Table 1). 
Table 2: Cell Dry Weight (CDW), Total Fatty Acids (TFA) and SDA yields in control strains

\begin{tabular}{|c|c|c|c|}
\hline Cultivation time(hour) & $\mathrm{CDW}(\mathrm{g} / \mathrm{l})$ & TFA (g/l) & $\mathrm{SDA}(\mathrm{mg} / \mathrm{l})$ \\
\hline \multicolumn{4}{|l|}{ Mc-2079 } \\
\hline $12 \mathrm{~h}$ & $9.70 \pm 0.77$ & $1.17 \pm 0.64$ & $100 \pm 0.43$ \\
\hline $24 \mathrm{~h}$ & $18.7 \pm 0.7$ & $4.30 \pm 0.44$ & $320 \pm 0.47$ \\
\hline $36 \mathrm{~h}$ & $19.21 \pm 0.65$ & $5.39 \pm 0.91$ & $300 \pm 0.46$ \\
\hline $48 \mathrm{~h}$ & $19.8 \pm 0.4$ & $6.39 \pm 0.52$ & $330 \pm 0.75$ \\
\hline $60 \mathrm{~h}$ & $19.9 \pm 0.38$ & $6.90 \pm 0.67$ & $340 \pm 0.80$ \\
\hline $72 \mathrm{~h}$ & $19.7 \pm 0.7$ & $6.81 \pm 0.16$ & $320 \pm 1.00$ \\
\hline $84 \mathrm{~h}$ & $19.3 \pm 0.43$ & $6.70 \pm 0.03$ & $290 \pm 0.75$ \\
\hline $96 \mathrm{~h}$ & $19 \pm 0.5$ & $6.40 \pm 0.23$ & $270 \pm 0.35$ \\
\hline
\end{tabular}

The values represent the mean \pm SD of three independent experiments

\section{Discussion}

In our present study, the high lipid accumulating strain $M$. circinelloides WJ11 was used to construct an SDA producing cell factory by overexpression of $\mathrm{fad} 3$ ( $\omega$-3 desaturase) gene of $A$. thaliana, which is reported to convert LA to ALA (Mendes et al., 2013) and also GLA to SDA. In M. circinelloides WJ11 we constructed the complete metabolic pathway by overexpression of fad3 gene (Fig. 1). The other gene, D6D, was already present in this fungus (Sakuradani et al., 2008). The accumulation of preferred products such as the biosynthesis of Very-Long-Chain Polyunsaturated Fatty Acid (VLCPUFA) in recombinant microbes can be further improved by more research (Sakuradani et al., 2008). Previously, we overexpressed Delta-15 Desaturase $(D 15 D)$ in the low lipid producing $M$. circinelloides CBS 277.49 strain where SDA yield was $64 \mathrm{mg} / \mathrm{L}$. The $D 15 D$ gene was cloned from another filamentous fungus Mortierella alpina (Khan et al., $2019 \mathrm{c}$ ). This time after overexpression of $\mathrm{fad} 3$ gene in M. circinelloides WJ11, the SDA yield was $340 \mathrm{mg} / \mathrm{L}$ which was the much better from our previous result.

Overexpression of $\mathrm{fad} 3$ gene did not affect the metabolic pathway to produce major fatty acids by $M$. circinelloides WJ11. The fatty acids, 16:0, 18:0, 18:1, 18:2 (LA) and 18:3 (GLA), were found as the major lipids before and after overexpression of this gene (Khan et al., 2019b; Yazawa et al., 2007; Lee et al., 2019). The natural sources, such as the plants, algae and fungi, produce SDA as a metabolic intermediate during the biosynthesis of long chain $\omega-3$ fatty acids; It was not found to be accumulated substantially in any species (Whelan, 2009). In the recombinant $M$. circinelloides $\mathrm{WJ} 11$, we confirmed the recombination and overexpression of $\mathrm{fad} 3$ gene in three ways: firstly, we checked its DNA, Secondly, by doing RT-qPCR which confirmed that the mRNA transcript level was kept elevated during the whole fermentation process and thirdly, by doing fatty acid profile analysis that showed the accumulation of SDA in this fungus. In Mc-2079, both ALA and SDA accumulated in significant amount which ultimately increased the total yield of $\omega-3$ fatty acids. In this metabolic pathway SDA occurred as the end product. M. circinelloides has been used for GLA production industrially for more than 30 years (Yazawa et al., 2007), so it can also be used for industrial production of SDA.

SDA production in plant sources is still in progress in comparison to microbial origin. Scientists cloned the D6D gene from Phytophthora citrophthora and overexpressed in Perilla frutescens and the accumulation of GLA and SDA was over 45\% (Lee et al., 2019). But the unavailability of genomic information kept scientists apart to understand the molecular basis of SDA accumulation in this plant (Sreedhar et al., 2017). Because of nutritional value and increased demand, researches are carried out worldwide to construct genetically modified SDA producing microbes. In Japan, Saccharomyces cerevisiae which was improved by gene manipulation could produce up to $13 \%$ SDA was constructed. But in that process the cultivation media was supplied with $0.8 \mathrm{~g} / \mathrm{L}$ histidine (Kimura et al., 2014; 2009). Our recombinant $M$. circinelloides WJ11 can produce almost $340 \mathrm{mg} / \mathrm{L} \mathrm{SDA}$ without any supplement. However, for the purpose of using $M$. circinelloides WJ11 as cell factory for industrial production SDA, further upscaling and optimization are required.

\section{Conclusion}

The recombinant SDA producing strain of $M$. circinelloides WJ11 constructed successfully by overexpression of $\mathrm{fad} 3$ gene of $A$. thaliana in our research may have applications for the commercial production of SDA. However, more research can be carried out for further upscaling and optimization of industrial cultivation of this recombinant $M$. circinelloides WJ11.

Abbreviations: $\quad$ M. circinelloides: Mucor circinelloides, A. thaliana: Arabidopsis thaliana, SDA: Stearidonic acid, LA: Linoleic acid, ALA: Alpha linolenic acid, GLA: Gamma linolenic acid, ARA: Arachidonic acid, EPA: Eicosapentaenoic acid, DHA: Docosahexaenoic acid, PUFA: Polyunsaturated fatty acid, VLCPUFA: Very-long-chain polyunsaturated fatty acid, D6D: Delta-6 Desaturase, D15D: Delta-15 
desaturase, GC: Gas chromatography, CDW: Cell dry weight, SAFA: Saturated fatty acids, MUFA: Monounsaturated fatty acids, TFA: Total fatty acids.

\section{Acknowledgment}

We are grateful to Muhammad Hafiy Bin Mohd Halim for his help in drawing pictures and analyzing the data.

Funding Information: This work was supported by the National Natural Science Foundation of China (Grant Nos. 31670064 and 31972851), Natural Science Foundation of Shandong province (ZR2018BC058) Science and Technology Project of Shandong College (J16LE20 and J17KA133), Taishan Industry Leading Talent Project and starting grant from Shandong University of Technology.

\section{Author Contributions}

Junhuan Yang and Md. Ahsanul Kabir Khan: Worked in the experimental design, fermentation testing, manuscript writing and figures and tables arrangement and contributed equally to this paper as first authors.

Sergio López-García: Built the MU760 strain which was the auxotroph of Mucor circinelloides WJ11.

Shaista Nosheen, Yusuf Nazir and Huaiyuan Zhang: Was involved in the experimental design.

Victoriano Garre: Carried out results interpretation, manuscript writing and reviewed of final draft.

Yuanda Song: Proposed the project and was involved in data analysis, result interpretation, manuscript writing and review of the final draft.

\section{Ethics Approval and Consent to Participate}

The authors did not perform any study with human participants or animals.

\section{Availability of Data and Materials}

All data generated and/or analyzed in this research are included in this published article [and its Additional file].

\section{Conflict of Interest}

There are no conflicts of interest in relation to this article.

\section{References}

Abeywardena, M.Y., M. Adams, J. Dallimore and S.M. Kitessa, 2016. Rise in DPA following SDA-rich dietary echium oil less effective in affording antiarrhythmic actions compared to high DHA levels achieved with fish oil in Sprague-Dawley rats. Nutrients, 8: 14-14. DOI: 10.3390/nu8010014
Ander, B.P., C.M.C. Dupasquier, M.A. Prociuk and G.N. Pierce, 2003. Polyunsaturated fatty acids and their effects on cardiovascular disease. Exp. Clin. Cardiol.

Baker, E.J., E.A. Miles, G.C. Burdge, P. Yaqoob and P.C. Calder, 2016. Metabolism and functional effects of plant-derived omega-3 fatty acids in humans. Prog. Lipid Res., 64: 30-56. DOI: $10.1016 /$ j.plipres.2016.07.002

Bilgiç, S. and N.Ş. Yeşilçubuk, 2012. Lipase-catalyzed acidolysis of olive oil with Echium oil stearidonic acid: Optimization by response surface methodology. JAOCS, J. Am. Oil Chem. Soc., 89: 1971-1980. DOI: 10.1007/s11746-012-2097-8

Chaney, A.L. and E.P. Marbach, 1962. Modified reagents for determination of urea and ammonia. Clin. Chem., 8: 130-132. DOI: $10.1093 /$ clinchem/8.2.130

Fan, Y.Y. and R.S. Chapkin, 1998. Importance of dietary $\gamma$-linolenic acid in human health and nutrition. J. Nutr., 128: 1411-1414. DOI: 10.1093/jn/128.9.1411

Harris, W.S., S.L. Lemke, S.N. Hansen, D.A. Goldstein and M.A. DiRienzo et al., 2008. Stearidonic acidenriched soybean oil increased the omega-3 index, an emerging cardiovascular risk marker. Lipids, 43: 805-811. DOI: 10.1007/s11745-008-3215-0

Horrobin, D.F., 1992. Nutritional and medical importance of gamma-linolenic acid. Prog. Lipid Res., 31: 163-194. DOI: 10.1016/0163-7827(92)90008-7

Kendrick, A. and C. Ratledge, 1992. Desaturation of polyunsaturated fatty acids in Mucor circinelloides and the involvement of a novel membrane-bound malic enzyme. Eur. J. Biochem., 209: 667-673. DOI: 10.1111/j.1432-1033.1992.tb17334.X

Khan, M.A.K., J. Yang, S.A. Hussain, H. Zhang and L. Liang et al., 2019a. Construction of DGLA producing cell factory by genetic modification of Mucor circinelloides. Microb. Cell Fact.

Khan, M.A.K., J. Yang, S.A. Hussain, H. Zhang and V. Garre et al., 2019b. Genetic modification of mucor circinelloides to construct stearidonic acid producing cell factory. Int. J. Mol. Sci., 20: 1683-1683. DOI: 10.3390/ijms20071683

Khan, M.A.K., J. Yang, S.A. Hussain, H. Zhang and V. Garre et al., 2019c. Genetic modification of mucor circinelloides to construct stearidonic acid producing cell factory. Int. J. Mol. Sci.

Kim, D.H., T.H. Yoo, S.H. Lee, H.Y. Kang and B.Y. Nam et al., 2012. Gamma linolenic acid exerts antiinflammatory and anti-fibrotic effects in diabetic nephropathy. Yonsei Med. J., 53: 1165-1165. DOI: $10.3349 / \mathrm{ymj} .2012 .53 .6 .1165$ 
Kimura, K., N. Tomita, H. Uemura, T. Aki and K. Ono et al., 2009. Improvement of stearidonic acid production in oleaginous Saccharomyces cerevisiae. Biosci. Biotechnol. Biochem., 73: 1447-1449. DOI: 10.1271/bbb.90082

Kimura, K., Y. Kamisaka, H. Uemura and M. Yamaoka, 2014. Increase in stearidonic acid by increasing the supply of histidine to oleaginous Saccharomyces cerevisiae. J. Biosci. Bioeng., 117: 53-56.

DOI: 10.1016/j.jbiosc.2013.06.004

Lee, K.R., K.H. Kim, J.B. Kim, S.B. Hong and I. Jeon et al., 2019. High accumulation of $\gamma$ linolenic acid and Stearidonic acid in transgenic Perilla (Perilla frutescens var. frutescens) seeds. BMC Plant Biol., 19: 120-120

DOI: $10.1186 / \mathrm{s} 12870-019-1713-2$

Lu, H. and Y. Zhu, 2015. Screening and molecular identification of overproducing $\gamma$-linolenic acid fungi and cloning the delta 6-desaturase gene. Biotechnol. Applied Biochem., 62: 316-322. DOI: $10.1002 /$ bab. 1281

Mendes, A., A.A. Kelly, H. van Erp, E. Shaw and S.J. Powers et al., 2013. bZIP67 regulates the omega-3 fatty acid content of Arabidopsis seed oil by activating fatty acid DESATURASE3. Plant Cell.

Petrik, M.B.H., M.F. McEntee, B.T. Johnson, M.G. Obukowicz and J. Whelan, 2000. Highly unsaturated (n-3) fatty acids, but Not $\alpha$-linolenic, conjugated linoleic or $\gamma$-linolenic acids, reduce tumorigenesis in ApcMin/+ Mice. J. Nutr. [Internet]. 130: 2434-2443.

DOI: $10.1093 / \mathrm{JN} / 130.10 .2434$

Rodríguez-Frómeta, R.A., A. Gutiérrez, S. TorresMartínez and V. Garre, 2013. Malic enzyme activity is not the only bottleneck for lipid accumulation in the oleaginous fungus Mucor circinelloides. Applied Microbiol. Biotechnol., 97: 3063-3072. DOI: 10.1007/s00253-012-4432-2
Sakuradani, E., S. Murata, H. Kanamaru and S. Shimizu, 2008. Functional analysis of a fatty acid elongase from arachidonic acid-producing Mortierella alpina 1S-4. Applied Microbiol. Biotechnol., 81: 497-503. DOI: $10.1007 / \mathrm{s} 00253-008-1675-\mathrm{z}$

Sreedhar, R.V., P. Prasad, L.P.A. Reddy, R. Rajasekharan and M. Srinivasan, 2017. Unravelling a stearidonic acid-rich triacylglycerol biosynthetic pathway in the developing seeds of Buglossoides arvensis: A transcriptomic landscape. Sci. Rep.

Tang, X., X. Zan, L. Zhao, H. Chen and Y.Q. Chen et al., 2016. Proteomics analysis of high lipid-producing strain Mucor circinelloides WJ11: An explanation for the mechanism of lipid accumulation at the proteomic level. Microb. Cell Fact.

Wang, C., W.S. Harris, M. Chung, A.H. Lichtenstein and E.M. Balk et al., 2006. n-3 Fatty acids from fish or fish-oil supplements, but not $\alpha$-linolenic acid, benefit cardiovascular disease outcomes in primaryand secondary-prevention studies: A systematic review. Am. J. Clin. Nutr., 84: 5-17.

Wang, M., J. Chen, H. Zhang and Y. Song, 2014. Molecular switch that controls the flux of linoleic acid into N-6 or N-3 polyunsaturated fatty acids in microorganisms. Am. J. Biochem. Biotechnol., 10: 105-115. DOI: 10.3844/ajbbsp.2014.105.115

Whelan, J., 2009. Dietary stearidonic acid is a long chain (n-3) polyunsaturated fatty acid with potential health benefits. J. Nutr., 139: 5-10. DOI: $10.3945 /$ jn. 108.094268

Yazawa, H., H. Iwahashi, Y. Kamisaka, K. Kimura and T. Aki et al., 2007. Heterologous production of dihomo- $\gamma$-linolenic acid in Saccharomyces cerevisiae. Applied Environ. Microbiol.

Zhang, Y., X. Luan, H. Zhang, V. Garre and Y. Song et al., 2017. Improved $\gamma$-linolenic acid production in Mucor circinelloides by homologous overexpressing of delta-12 and delta- 6 desaturases. Microb. Cell Fact. 